\title{
HEPATECTOMIA LAPAROSCÓPICA COM PORTAL ÚNICO: EXPERIÊNCIA PIONEIRA NO BRASIL
}

\author{
First single-port laparoscopic liver resection in Brazil
}

\author{
Marcel Autran Cesar MACHADO, Rodrigo Cañada Trofo SURJAN, Fábio Ferrari MAKDISSI
}

Trabalho realizado no Hospital Sírio-Libanês, São Paulo, SP, Brasil.

DESCRITORES - Fígado. Técnicas. Laparoscopia. Hepatectomia. Portal único.

\section{Correspondência: \\ Marcel Autran Cesar Machado \\ E-mail: dr@drmarcel.com.br \\ Fonte de financiamento: não há \\ Conflito de interesses: não há}

Recebido para publicação: 04/02/2013 Aceito para publicação: 11/03/2013

HEADINGS - Liver. Techniques. Laparoscopy. Hepatectomy. Single-port.
RESUMO - Introdução - Na última década, a cirurgia laparoscópica evoluiu e hoje produz menor trauma graças à redução do número e tamanho dos trocárteres. Recentemente nova técnica com uso de portal único foi descrita. Objetivo Descrever os detalhes de uma segmentectomia lateral esquerda (segmentos 2 e 3) laparoscópica com portal único. Técnica - Portal único com cobertura de gel é introduzido por meio de incisão de $3 \mathrm{~cm}$ periumbilical. O procedimento inicia-se com exploração da cavidade com laparoscópio e exame ultra-sonográfico do fígado. É realizado Acesso intra-hepático ao pedículo Glissoniano dos segmentos 2 e 3 . O pedículo é seccionado com grampeador com carga vascular. O fígado é seccionado com bisturi harmônico e a veia hepática esquerda é dividida com grampeador. A peça é retirada pelo portal único. A cavidade é revista mas não é deixado dreno. Conclusão - Segmentectomia lateral esquerda laparoscópica com portal único é procedimento seguro desde que realizado em centros especializados e por equipes com experiência em cirurgia hepática e laparoscopia avançada.

ABSTRACT - Introduction - Since the past decade, minimal access surgery is moving towards minimizing the surgical trauma by reducing numbers and size of the laparoscopic ports. A novel technique with a single-incision laparoscopic approach has been recently described. Aim - To describe the single-port technique for laparoscopic liver resection. Technique - A transumbilical 3-cm skin incision is performed and a single-incision advanced access platform is introduced. Operation began with exploration of the abdominal cavity and ultrasound examination of the liver. Intrahepatic Glissonian access for retrieval of portal pedicles from segments 2 and 3 is performed. Vascular endoscopic stapler is used to divide segments 2 and 3 Glissonian pedicle. Liver is transected with harmonic scalpel and left hepatic vein is divided with stapler. Procedure is completed. Surgical specimen is retrieved through the single umbilical incision. No drains are left in place. Conclusion - Single port laparoscopic left lateral segmentectomy is feasible and can be safely performed in specialized centers by skilled laparoscopic surgeons.

\section{INTRODUÇÃO}

$\mathrm{N}$ a última década, a cirurgia minimamente invasiva vem evoluindo progressivamente com redução do trauma cirúrgico graças à redução do número e tamanho dos trocárteres. Nos últimos anos, foi descrita uma nova técnica laparoscópica com uso de incisão única 5 . Ela técnica é muito utilizada em mulheres e jovens em virtude do bom resultado cosmético. No Brasil e em outros países esta técnica tem sido empregada geralmente em colecistectomia laparoscópica ${ }^{12}$.

A cirurgia hepática e o manejo ds doenças do fígado vem apresentando grande evolução. Hoje a hepatectomia laparoscópica é realidade no nosso meio e estes autores a têm empregado de maneira rotineira desde 2007 com mais de 100 casos descritos, incluindo ressecções maiores ${ }^{8,9,10,11}$.

O objetivo deste trabalho é descrever a hepatectomia laparoscópica usando a técnica de portal único. Neste relato, descreve-se os aspectos técnicos de uma hepatectomia lateral esquerda (segmentos 2 e 3) laparoscópica com portal único. Do nosso conhecimento, trata-se do primeiro caso de hepatectomia com portal único no Brasil e América Latina. 


\section{TÉCNICA}

O paciente é colocado em decúbito dorsal e proclive com cirurgião entre as pernas. $O$ assistente posiciona-se no lado direito com o monitor o acima da cabeça do paciente. Sob anestesia geral, é realizada uma incisão transumbilical de $3 \mathrm{~cm}$. Por ela é introduzida um sistema de portal único com gel (GelPoint, Applied Med. RS Margarita, CA, EUA) (Figura 1a). Três portais de trabalho $(5-10 \mathrm{~mm})$ são introduzidos no dispositivo. Não há necessidade de uso de instrumentos articulados. O pneumoperitônio de $\mathrm{CO}_{2}$ é estabelecido a $12 \mathrm{~mm} \mathrm{Hg}$. Usa-se laparoscópio rígido de 30 o de 10 milímetros. A plataforma de portal único é capaz de acomodar simultaneamente três ou quatro instrumentos sem prejuízo de triangulação, dependendo da configuração necessária (Figura $1 \mathrm{~b}$ ). Dois grandes instrumentos, tais como um laparoscópio de $10 \mathrm{~mm}$, um grampeador flexível de $12 \mathrm{~mm}$ podem ser utilizados simultaneamente com um instrumento de $5 \mathrm{~mm}$. Outra configuração possível é o uso de uma câmera de $10 \mathrm{~mm}$ junto com mais quatro instrumentos de $5 \mathrm{~mm}$, porque a incisão inicial e abertura aponeurose possui $30 \mathrm{~mm}$ de largura. O procedimento inicia-se com exploração da cavidade abdominal com o laparoscópio e introdução do probe de ultrassom laparoscópico (SonoSite, Inc., Bothell, WA, EUA) para avaliar o tumor e sua relação com estruturas anatômicas hepáticas (Figuras 1c, 1d, 1e). Após esta avaliação inicial, o fígado esquerdo é mobilizado por meio de secção dos ligamentos falciforme, triangular e coronário com bisturi harmônico (Ultracision, Ethicon Endo-Surgery, Cincinnati, OH). O lobo esquerdo é tracionado para cima, e o omento menor é aberto.

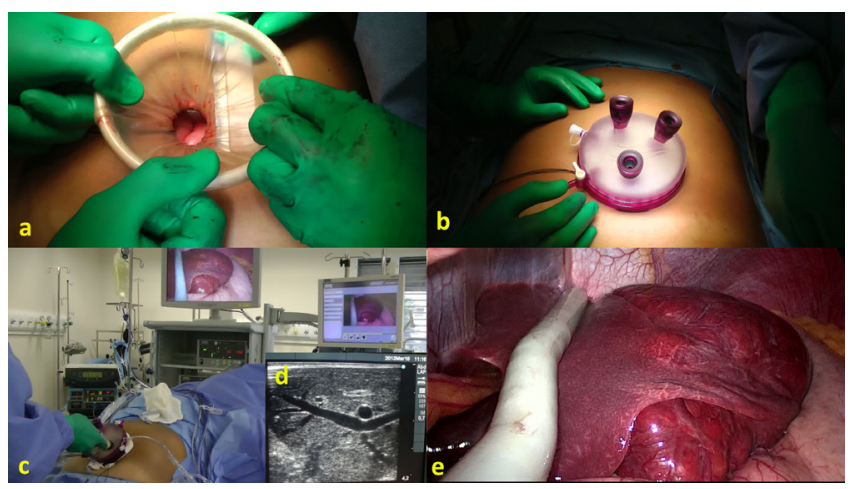

FIGURA 1 - Instalação do portal único: a) após incisão de $3 \mathrm{~cm}$ na pele ao redor do umbigo e abertura da aponeurose, o sistema de portal único é inserido; b) é realizada cobertura de gel já com três portais de trabalho (5 a $11 \mathrm{~mm}$ ) instalados; c) ultrassonografia intra-operatória é realizada através do portal único; uma camêra de alta definição $\left(30^{\circ}, 10 \mathrm{~mm}\right)$ é utilizada em conjunto com a sonda de ultrassonografia notando-se que não há necessidade de uso de laparoscópio de $5 \mathrm{~mm}$ ou instrumentos flexíveis; d) imagem ultrassonográfica mostra o tumor e a anatomia hepática; e) visualização interna do fígado e da sonda de ultrassonografia.
O próximo passo é a realização do acesso intrahepático ao pedículo Glissoniano dos segmentos 2 e 3. Duas incisões são necessárias. A primeira é feita acima da extremidade proximal do ligamento de Arantius e a segunda na base esquerda do ligamento redondo ${ }^{11}$. Uma pinça vascular é introduzida através destas incisões e fechada. Depois de alguns minutos, é observada a delimitação isquêmica de segmentos 2 e 3. A futura linha de transecção é marcada na cápsula hepática com cautério. A pinça vascular é substituída por grampeador endoscópico (Figura 2a). Um grampeador é disparado e é seccionado o pedículo portal dos segmentos 2 e 3 (Figura 2b). A transecção hepática é feita com bisturi harmônico. O último passo é seccionar a veia hepática esquerda com grampeador. O procedimento é concluído e o espécime cirúrgico é colocado dentro de um saco plástico e retirado através da incisão umbilical. Dependendo do tamanho do espécime cirúrgico, a incisão tem de ser alargada, mas geralmente o segmento lateral esquerdo pode ser retirado através da incisão umbilical de $3 \mathrm{~cm}$. A plataforma de portal único é recolocada para verificar a existência de sangramento e fístula biliar na superfície cruenta do fígado. Tecido hemostático é aplicado nessa superfície do fígado e a operação é finalizada (Figura 2c). A cavidade não é drenada.

Foi realizado este procedimento em uma mulher de 33 anos de idade, com adenoma de fígado. A duração do procedimento foi de 54 minutos. O sangramento foi mínimo, sem necessidade de transfusão e ela recebeu alta hospitalar no primeiro dia do pós-operatório. O aspecto cosmético da incisão foi bom após o procedimento (Figura 2d).

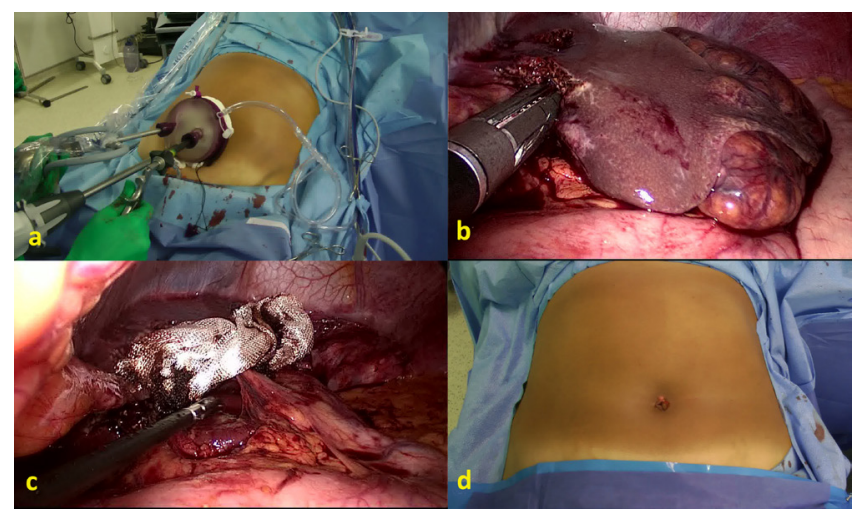

FIGURA 2 - Aspectos técnicos da segmentectomia lateral esquerda laparoscópica com portal único: a) vista externa da plataforma de portal único com ótica de $10 \mathrm{~mm}$, pinça vascular de 10 $\mathrm{mm}$ e grampeador endoscópico com $12 \mathrm{~mm}$ no mesmo portal; b) vista interna mostrando pedículo Glissoniano ocluído pelo grampeador fechado resultando em delimitação isquêmica do segmento lateral esquerdo (segmentos 2 e 3); c) visuzalização interna mostrando a operação concluída; d) imagem externa mostrando aspecto final da incisão umbilical. 


\section{DISCUSSÃO}

A cirurgia laparoscópica tornou-se uma excelente alternativa menos invasiva à cirurgia de fígado. Avanços de imagem pré e intra-operatório resultaram em uma melhor avaliação anatômica e, assim, melhor planejamento cirúrgico.

A cirurgia hepática laparoscópica tem experimentado um desenvolvimento significativo nos últimos anos. Nossa experiência com ressecções hepáticas laparoscópicas começou em 2007. Da mesma forma como ocorreu em outros centros, o aumento da experiência em cirurgia laparoscópica avançada nos permitiu realizar operações mais complexas, como a hepatectomia direita, em dois tempos e mesmo ALPPS totalmente laparoscópico ${ }^{8,9,10,11}$.

No entanto, apenas alguns centros, incluindo o nosso, realizaam a ressecção hepática laparoscópica de rotina ${ }^{13}$. Para vários autores, apenas segmentectomia esquerda laparoscópica lateral é considerado padrãoouro $^{3}$. Para nós, qualquer cirurgia hepática anatômica pode ser feito por laparoscopia, mas estamos de acordo que só devem ser feito em centros especializados e por equipes capacitadas ${ }^{8,13}$.

A cirurgia laparoscópica com portal único é menos invasiva do que o padrão com vários trocartes, mas existem dificuldades inerentes a este método ${ }^{14,15}$. Primeiro, a exposição é significativamente limitada. A introdução de uma câmara e vários instrumentos paralelos uns aos outras pode resultar numa diminuição da amplitude de movimento e na colisão de instrumentos ${ }^{1,2,4}$. A plataforma de portal único utilizada no nosso caso, permite o uso de instrumentos normais, sem perda de triangulação. Fomos capazes de utilizar uma ótica de $10 \mathrm{~mm}$ de alta definição, durante todos os passos da operação.

Optamos por realizar uma segmentectomia esquerda lateral laparoscópica por portal único, porque este procedimento é a hepatectomia mais fácil de realizar por laparoscopia e sua técnica já está padronizada ${ }^{3}$.

$\mathrm{Na}$ literatura existem poucos trabalhos de ressecção hepática com portal único ${ }^{6,7,14,15}$. A principal razão é que a maioria dos sistemas disponíveis para o uso desta técnica necessita de instrumentos especiais com articulação e uso de laparoscópios pequenos com triangulação reduzida. Acreditamos que este novo sistema com gel pode oferecer uma plataforma melhor para esta técnica e pode reduzir a curva de aprendizagem. Na nossa experiência inicial com um segmentectomia lateral esquerda laparoscópica com portal único não houve nenhuma dificuldade de exposição ou de triangulação e o tempo operatório não foi superior aos nossos outros casos de laparoscopia. Do nosso conhecimento, trata-se do primeiro relato do uso desta técnica em cirurgia do fígado laparoscópica no Brasil e na América Latina.

Apesar do custo e da curva de aprendizado,os benefícios cosméticos da cirurgia com portal único são óbvios. Em conclusão, segmentectomia lateral esquerda laparoscópica com portal único é viável e pode ser realizada com segurança em centros especializados e por cirurgiões laparoscópicos capacitados.

\section{REFERÊNCIAS}

1. Aikawa M, Miyazawa M, Okamoto K, Toshimitsu Y, Okada K, Ueno Y, Yamaguchi S, Koyama I. Single-port laparoscopic hepatectomy: technique, safety, and feasibility in a clinical case series. Surg Endosc 2012; 26:1696-1701.

2. Aldrighetti L, Guzzetti E, Ferla G. Laparoscopic hepatic left lateral sectionectomy using the LaparoEndoscopic Single Site approach: evolution of minimally invasive liver surgery. J Hepatobiliary Pancreat Sci. 2011;18:103-5.

3. Azagra JS, Goergen M, Brondello S, Calmes MO, Philippe P, Schmitz B. Laparoscopic liver sectionectomy 2 and 3 (LLS 2 and 3): towards the "gold standard". J Hepatobiliary Pancreat Surg 2009;16:422-6.

4. Chang SK, Mayasari M, Ganpathi IS, Wen VL, Madhavan K.Single port laparoscopic liver resection for hepatocellular carcinoma: a preliminary report. Int J Hepatol 2011;2011:579203.

5. Canes D, Desai MM, Aron M, Haber GP, Goel RK, Stein RJ, Kaouk $\mathrm{JH}$, Gill IS. Transumbilical single-port surgery: evolution and current status. Eur Urol 2008;54(5):1020-9

6. Dapri G, Dimarco L, Cadière GB, Donckier V. Initial experience in single-incision transumbilical laparoscopic liver resection: indications, potential benefits, and limitations. HPB Surg 2012;2012:921973.

7. Gaujoux $S$, Kingham TP, Jarnagin WR, D'Angelica MI, Allen PJ, Fong Single-incision laparoscopic liver resection. Surg Endosc 2011;25:1489-94. Y.

8. Machado MA, Makdissi FF, Surjan RC. Laparoscopic liver resection: personal experience with 107 cases. Rev Col Bras Cir 2012;39:483-8.

9. Machado MA, Makdissi FF, Surjan RC. Intrahepatic Glissonian approach for pure laparoscopic right hemihepatectomy. Surg Endosc. 2011; 25(12):3930-3.

10. Machado MA, Makdissi FF, Surjan RC. Totally laparoscopic ALPPS is feasible and may be worthwhile. Ann Surg 2012;256:e13.

11. Machado MA, Makdissi FF, Surjan RC, Herman P, Teixeira AR, C Machado MC. Laparoscopic resection of left liver segments using the intrahepatic Glissonian approach. Surg Endosc. 2009; 23:26152619.

12. Madureira FA, Manso JE, Madureira Fo D, Iglesias AC. Randomized clinical study for assessment of incision characteristics and pain associated with LESS versus laparoscopic cholecystectomy. Surg Endosc 2013;27:1009-15.

13. Nguyen KT, Gamblin TC, Geller DA. World review of laparoscopic liver resection-2,804 patients. Ann Surg. 2009;250:831-41.

14. Patel AG, Belgaumkar AP, James J, Sing UP, Carswell KA, Murgatroyd B. Single-incision laparoscopic left lateral segmentectomy of colorectal liver metastasis. Surg Endosc 2011;25:649-50.

15. Shetty GS, You YK, Choi HJ, Na GH, Hong TH, Kim DG. Extending the limitations of liver surgery: outcomes of initial human experience in a high-volume center performing single-port laparoscopic liver resection for hepatocellular carcinoma. Surg Endosc 2012;26:1602-8. 